\title{
Aktueller Stand der Stammzelltherapie in Orthopädie und Unfallchirurgie
}

\section{Stem Cell-Based Therapy in Orthopaedics and Trauma Surgery - Current Concepts}

Autoren

Institute
T. M. Randau ${ }^{1}$, S. Gravius ${ }^{1}$, A. Nüssler ${ }^{2}$

${ }^{1}$ Klinik und Poliklinik für Orthopädie und Unfallchirurgie, Universitätsklinikum Bonn

${ }^{2}$ Siegfried Weller Institut für Unfallmedizinische Forschung, BG Unfallklinik, Eberhard-Karls Universität Tübingen

\author{
Schlüsselwörter \\ - Stammzellen \\ - Tissue Engineering \\ - Knochen \\ - Knorpel \\ Key words \\ - stem cells \\ - tissue engineering \\ - bone \\ - cartilage
}

\section{Zusammenfassung \\ $\nabla$}

In den letzten Jahren wurden viele neue Heilverfahren für muskuloskeletale Erkrankungen entwickelt. Einige dieser neuen Verfahren beruhen auf dem gezielten Einsatz von Stammzellen, um Heilungsprozesse zu initiieren, Defizite auszugleichen oder die Regeneration von Sehnen, Muskeln, Knochen oder Knorpel zu aktivieren. Dies kann durch den direkten Einsatz von Stammzellen auf oder in einem Trägermaterial oder durch eine Kombination mit Tissue Engineering erreicht werden. In diesem Artikel geben wir einen kurzen Überblick über mögliche Einsatzgebiete von induzierbaren pluripotenten, hämatopoetischen und adulten Stammzellen sowie über deren Einsatz in muskuloskeletalem Gewebe. Zusätzlich fassen wir die derzeitige rechtliche Situation bei der Anwendung von Stammzellen am Menschen zusammen.

\section{Abstract \\ $\nabla$}

Over the last few years, numerous new treatment methods have been developed for musculoskeletal diseases. Some of these new methods are based on the targeted use of stem cells to initiate healing processes, to compensate for deficits or to activate the regeneration of tendons, muscles, bones and cartilage. This goal can be achieved through the direct use of stem cells on or in a carrier material or through a combination with tissue engineering. In this article, we give a short overview of the possible fields of application of inducible pluripotent haematopoietic, and adult stem cells as well as on their use in musculoskeletal tissue. Furthermore, we provide a summary of the current legal situation concerning the application of stem cells in humans.

\section{Bibliografie}

Dol http://dx.doi.org/ 10.1055/s-0034-1382867 Z Orthop Unfall 2014; 152: 320-327 @ Georg Thieme Verlag KG Stuttgart · New York . ISSN 1864-6697

\section{Korrespondenzadresse} Dr. med. Thomas Randau Klinik und Poliklinik für Orthopädie und Unfallchirurgie Universitätsklinikum Bonn Sigmund-Freud-Straße 25 53105 Bonn

thomas.randau@ ukb.uni-bonn.de

\section{License terms}

\section{()(1) $\Theta$}

\section{Einleitung}

Muskuloskeletale Erkrankungen stellen die weltweit häufigste Ursache chronischer Schmerzen und dauerhafter Behinderungen dar. Sie betreffen hunderte Millionen Patienten und ihr medizinisches und sozioökonomisches Ausmaß ist enorm. Während bei älteren Patienten degenerative Erkrankungen die wesentliche Krankheitslast ausmachen, führen bei den jungen Patienten in erster Linie die Folgen von Unfällen und Verletzungen zu dauerhaften Behinderungen, Funktionsausfällen und zu Erwerbsunfähigkeit. Um dem steigenden Bedarf in der Patientenversorgung nicht nur medizinisch, sondern auch wissenschaftlich gegenüberzutreten, wurde das vergangene Jahrzehnt zur "Decade of the Bone and Joint“ ernannt [1]. Nicht zuletzt dadurch hat das Forschungsinteresse in diesem Bereich über die letzten Jahre hinweg stark zugenommen. Obwohl diese Dekade seit 2010 offiziell vorüber ist, setzt sich dieser
Trend weiter fort, und immer mehr Arbeitsgruppen widmen sich der Grundlagenforschung im Bereich der muskuloskeletalen Erkrankungen [2]. Heutzutage bietet das neu geschaffene Feld der regenerativen Medizin vielversprechende Lösungsansätze zur Behandlung von Problemen, die durch den Substanz- oder Funktionsverlust des Stütz- und Bewegungsapparats entstehen [3]. Im Rahmen des sog. „Tissue Engineering“, also der In-vitro-Züchtung von biologischem Organ- oder Gewebeersatz zur späteren Implantation, wird häufig vom sog. „Triangle Concept“ gesprochen. Dieser Begriff bezeichnet die Kombination der 3 Grundfaktoren des Tissue Engineering, also einem Trägermaterial (oder „Scaffold“), mehreren Wachstumsfaktoren und den Stammzellen. Daneben wird auch die alleinige Anwendung von stammzellhaltigen Zellsuspensionen, Knochenmarkkonzentrationen oder isolierten und aufgereinigten Stammzellen zunehmend experimentell untersucht. 


\section{Die verschiedenen Stammzelltypen}

$\nabla$

Der Begriff „Stammzelle“ umfasst mehrere Arten von Zellen, die aus unterschiedlichen Herkunftsgeweben stammen und bez. ihres regenerativen Potenzials variieren. Daher ist eine genauere Definition dieses Begriffs sowie die Vorstellung der Eigenschaften und Grenzen der unterschiedlichen Zelltypen, die unter diesem Begriff zusammengefasst werden, notwendig [4].

\section{Mesenchymale Stammzellen (MSCs)}

Die Regeneration aller Gewebe des Stütz- und Bewegungsapparats geht von einem gemeinsamen Pool an Vorläufer- oder Stammzellen aus. Gemäß ihrem Herkunftsgewebe und ihrem Potenzial zur Differenzierung in verschiedene Gewebearten wurden diese Zellen mit verschiedenen Bezeichnungen versehen. Alle verwendeten Bezeichnungen, wie z.B. „Bone marrow derived stem cells“ (BMSCs), „postnatale skeletale Stammzellen“ (SSCs), „mesenchymale Stammzellen“, „marrow stromal cells“ (MSCs) oder „multipotente adulte Vorläuferzellen“ [5,6], beschreiben Zellpopulationen, die einander zwar sehr ähnlich, aber dennoch nicht identisch sind. Daher muss an dieser Stelle festgehalten werden, dass es „die“ Stammzelle des Bewegungsapparats nicht gibt und dass alle adulten Stammzellpopulationen stets multiklonale Mischkulturen sind, die sich merklich voneinander unterscheiden [7].

Die mesenchymalen Stammzellen wurden erstmals im Jahre 1966 von Friedenstein et al. in einem Isolat aus dem Knochenmark von Ratten identifiziert. In diesem Isolat fanden die Forscher Zellen, welche sich anhaltend vermehren konnten und sich zu knochenbildenden Zellen differenzieren ließen [8]. Doch die MSCs verfügen über weitere Eigenschaften, die sie zu einem interessanten Forschungsobjekt im Bereich der regenerativen Medizin machen [9]. So lassen sie sich bspw. unter definierten Bedingungen in verschiedene Gewebearten differenzieren, darunter Knochen, Fett, Knorpel, Bindegewebe und Muskulatur [10,11], sind aber als multipotente Zellen in ihrer Differenzierung bereits weitgehend (jedoch vermutlich nicht exklusiv) auf mesenchymale Gewebe festgelegt. Sie kommen im Stroma fast aller Gewebe sowie im peripheren Blut vor, jedoch werden sie hauptsächlich aus dem Knochenmark isoliert. Obwohl sie im Knochenmark nur einen sehr geringen Anteil der kernhaltigen Zellen ausmachen (0,01-0,001\%), können sie dank ihrer Adhärenz an Zellkulturplastik, ihrer Fähigkeit zur Selbsterneuerung und ihrer proliferativen Aktivität relativ einfach und mit hoher Effizienz isoliert und expandiert werden. MSCs unterliegen zumindest in Kultur einer „Seneszenz“, da ihre Proliferation nach einigen Passagen zum Erliegen kommt und sie nach protrahierter Kultur ihre Differenzierungsfähigkeit verlieren.

Um MSCs von anderen Stromazellen und von hämatopoetischen Stammzellen des Knochenmarkes sicher unterscheiden zu können, wurden verschiedene Oberflächenmarker (sog. „Clusters of Differentiation“, CDs) definiert. MSCs fehlt der hämatopoetische Marker „Protein Tyrosin Phosphatase Rezeptor Typ C“ (PTPRC, CD45), sie weisen jedoch das, ebenfalls mit dem Endothelgewebe assoziierte, „Interzelluläre Adhäsionsmolekül 1“ (ICAM-1, CD54) sowie das aktivierte „Leukozyten-Zell-Adhäsionsmolekül“ (ALCAM, CD166) [12] und den Wachstumsfaktorrezeptor „Endoglin“ (CD105) auf [13]. Weitere typische Oberflächenmarker sind u.a. CD29, CD44, CD73 und CD90. Diese Marker sind so spezifisch, dass einige von ihnen, neben der Plastikadhärenz und der Differenzierbarkeit, zu den „Minimalanforderungen“ an MSCs gezählt werden [14]. MSCs verfügen außerdem über die Fähigkeit, Zellen des Immunsystems zu regulieren und auf diese Weise die
Immunantwort lokal zu unterdrücken [15]. Sie verfügen über die Rezeptoren für zahlreiche Zytokine und können mit anderen Zellen des Immunsystems parakrin kommunizieren [16]. Diese Eigenschaft ermöglicht nicht nur ihren Einsatz als Therapeutikum bei Autoimmunerkrankungen, sondern ist auch von besonderer Bedeutung für die Frakturheilung, wo erst durch das Einwandern von MSCs die inflammatorische Phase in die regenerative Phase übergehen kann $[17,18]$.

\section{Embryonale Stammzellen (ESCs)}

MSCs bleiben, wenn auch in abnehmender Zahl, im Organismus lebenslang verfügbar und werden daher als „adulte“ Stammzellen bezeichnet. Ihnen gegenüber stehen die embryonalen Stammzellen (ESCs). Die pluripotenten oder gar omnipotenten ESCs wurden erstmalig 1981 aus Mausembryonen gewonnen. Im Jahre 1998 wurden sie dann erstmals aus menschlichen Embryonen isoliert. Die ESCs sind in ihrer Differenzierung noch nicht festgelegt und können sich noch in die 3 Zellschichten Ektoderm, Mesoderm und Endoderm differenzieren [19,20]. Sie proliferieren ohne Anzeichen einer Zellalterung und sind damit in Kultur quasi „unsterblich“. Auch für ESCs wurden, neben ihrer Herkunft sowie ihrer Proliferations- und Differenzierungskapazität, bestimmte Oberflächenmarker (SSEA3, SEA4, TRA-1-60, TRA-1-81 und ALP) als typische Erkennungsmerkmale definiert [20]. Dank dieser Eigenschaften stellen die ESCs einen vielversprechenden Zelltyp für die Anwendung in der gesamten regenerativen Medizin dar. Ihr Einsatz ist somit nicht auf den muskuloskeletalen Bereich beschränkt. Ihrer klinischen Anwendung stehen jedoch hauptsächlich juristische Probleme und ethische Vorbehalte gegen ihre Herkunft und ihre Herstellung aus potenziell implantierbaren und lebensfähigen menschlichen Embryonen im Wege. Zudem ist ein autologer Zelltransfer auf potenzielle Patienten unmöglich, da die Zellen in der frühesten Embryonalphase gewonnen werden müssen. Ein allogener Transfer birgt jedoch die aus der hämatopoetischen Stammzelltransplantation bekannten Schwierigkeiten bez. der Immunkompatibilität. Aktuell sind weitere Bemühungen zur Stabilisierung des Phänotyps von differenzierten ESCs notwendig, um das Risiko einer ungesteuerten De- und Redifferenzierung mit Bildung von Teratomen und malignen Tumoren weiter zu minimieren.

\section{Induzierte pluripotente Stammzellen (iPSCs)}

Die phänotypische Stabilität einer differenzierten Zelle im Organismus ist i.d. R. sehr hoch, aber dennoch nicht endgültig. Im Jahr 2006 gelang es erstmals, einen adulten Mausfibroblasten mittels spezifischer Transkriptionsfaktoren in Kultur auf das Stadium der Pluripotenz „zurückzusetzen“ [21]. Die so erzeugten Zellen haben als „induzierte pluripotente Stammzellen“ (iPSCs) seither sehr großes Forschungsinteresse geweckt. Kurze Zeit später konnte das Verfahren in modifizierter Form auch auf humane Zellen übertragen werden [22]. Die auf diese Art und Weise generierten iPSCs besitzen die typischen Oberflächenmerkmale von ESCs. Im Gegensatz zu letzteren können iPSCs jedoch patientenspezifisch, also autolog, generiert werden. Zudem ist die Entnahme der Ausgangszellen unkompliziert und ethisch kaum bedenklich. Inwieweit funktionelle Unterschiede zwischen beiden Zelltypen bestehen, wird aktuell noch untersucht [23]. Trotz Unterschieden auf Genebene, Proteinebene und in der Epigenetik, scheinen sich iPSCs und ESCs aber in den wesentlichen Funktionen Proliferation und Differenzierung weitgehend zu gleichen. Dies bringt jedoch für beide Zelltypen auch ähnliche Probleme in der phänotypischen Stabilisierung der Zellen mit sich. 
Während die Forschung an ESCs durch ethische und juristische Schwierigkeiten und ihre Anwendung durch immunologische Limitationen stark eingeschränkt ist, bieten iPSCs einen sehr vielversprechenden Ansatz für eine klinische Anwendung, sofern alle Sicherheitsbedenken gelöst werden können [24]. MSCs haben ihrerseits bereits den Sprung in die klinische Anwendung geschafft [25].

Trotz der Einführung von MSCs in die Klinik gilt es, eine Reihe von wissenschaftlichen Faktoren zu berücksichtigen. So hat sich bspw. herausgestellt, dass sich wahrscheinlich nicht jeder MSCZelltyp eines Organs gleichwertig in den jeweiligen gewünschten Zelltyp eines anderen Organs differenzieren lässt. Außerdem konnte gezeigt werden, dass MSCs einem Alterungsprozess unterliegen [26-28]. Insbesondere die MSCs älterer Patienten scheinen einen Alterungsprozess zu durchlaufen, der auch für die verzögerte Regeneration von Organen bei diesen Patienten verantwortlich sein könnte. So haben wir kürzlich zeigen können, dass 5-Azazytidine, ein DNA-Methyltransferase-Inhibitor (DNMT-Inhibitor), der zur Behandlung von Tumoren eingesetzt wird, nicht nur die Proliferation alter MSCs, sondern auch deren Pluripotenz verbessert und somit deren osteogene Differenzierung erhöht [29]. Auf diese Weise könnte der Heilungsprozess bei älteren Patienten verbessert bzw. beschleunigt werden.

\section{Anwendung am Knochen \\ $\nabla$}

\section{Frakturheilung und Pseudarthrosen}

Pseudarthrosen und gestörte Frakturheilung stellen einen potenziellen Anwendungsbereich für stammzellbasierte Therapien dar [30]. Der aktuelle Goldstandard für die Behandlung von Pseudarthrosen ist die autologe Spongiosa- oder Knochentransplantation, die letztlich nichts anderes als eine autologe Stammzelltransplantation im bestmöglichen Scaffold, nämlich dem eigenen Knochen, ist. Der Schritt zur Verwendung von Stammzellpräparaten in Form von Knochenmarkaspirat ist entsprechend naheliegend. Bereits im Jahre 1989 wurde eine Arbeit über die Verwendung von Knochenmarkaspirat zur Behandlung von Pseudarthrosen publiziert [31], und im Jahre 1991 wurde gezeigt, dass die Wirkung von Knochenmarkaspirat mit jener von autologer Spongiosa vergleichbar sein kann [32]. Im Jahre 2005 wurde im Rahmen einer Studie mit 60 Patienten die Wirksamkeit der Therapie atropher Pseudarthrosen der Tibia mittels perkutaner Injektion eines aufbereiteten Knochenmarkkonzentrats bewiesen [33]. Im Jahre 2009 kam eine randomisiert kontrollierte Studie mit 64 Patienten zu ähnlich guten Ergebnissen. Im Rahmen der letztgenannten Studie wurden die Zellen vor der Replantation ex vivo kultiviert und differenziert [34]. Auch in der primären Frakturheilung könnten MSCs gezielt injiziert werden, um eine Anreicherung von Knochenzellen im Frakturbereich zu erzielen und die Knochenheilung zu verbessern [35]. Analog zu großen Knochendefekten wäre auch bei der verzögerten Frakturheilung eine Kombination der Stammzellen mit Trägermaterialien und Wachstumsfaktoren sinnvoll und möglich, jedoch fehlt es an ausreichend guten und kontrollierten klinischen Studien, um die vermutete Wirksamkeit dieser Therapie endgültig beweisen zu können [36].

\section{Knochendefekte}

Aufgrund der geringen Anzahl an Behandlungsoptionen, liegt die Behandlung von Knochendefekten durch Tissue Engineering oder Stammzelltransplantationen nahe [37]. Nicht nur in der musku- loskeletalen Chirurgie, sondern auch in der Neurochirurgie und in der Mund-Kiefer-Gesichtschirurgie, stellen Knochenbrüche als Folge von Trauma, Tumor und Infektion, oder iatrogen nach gescheiterter Osteosynthese oder Gelenkersatz, eine klinische Herausforderung dar [38]. Auch in diesen Bereichen ist die autologe Spongiosaplastik der aktuelle Goldstandard. Die Menge an verfügbarem Knochen ist jedoch limitiert und die Entnahme komplikationsbehaftet [39]. Die als mögliche Alternative geltende Distraktionsosteogenese sieht sich jedoch mit den gleichen Schwierigkeiten konfrontiert [40]. Seit mehr als 10 Jahren werden nunmehr die Möglichkeiten und das hohe Potenzial der Stammzelltherapie und des stammzellbasierten Tissue Engineering für den Bereich der Knochendefekte propagiert [41]. Da die Biomechanik nun im Rahmen der Knochenheilung ebenfalls Berücksichtigung finden soll, wird aus dem klassischen „Triangle Concept“ das „Diamond Concept“ [42]. Neben sehr vielen Invitro-Studien wurden auch bereits zahlreiche Studien an verschiedenen Tiermodellen, Knochen und Scaffolds mit und ohne Wachstumsfaktoren, wie z.B. [43-46], publiziert. Die Anwendung am Menschen bleibt jedoch weiterhin auf eine geringe Anzahl an Fällen beschränkt, sodass kaum klinische Daten verfügbar sind. Wie mehrere vergleichende Untersuchungen bereits zeigen konnten, ist die Übertragbarkeit von Ergebnissen aus Tierversuchen in die klinische Praxis jedoch nur in sehr geringem Umfang möglich [47].

Die wahrscheinlich erste Anwendung des Knochen-Tissue-Engineering am Menschen wurde im Jahre 2001 von Quarto et al. durchgeführt [48]. Dabei wurde 4 Patienten im Rahmen einer kleinen Fallstudie ein mit Stammzellen besiedelter Hydoxylapatitzylinder (HA-Zylinder) implantiert. Diese Stammzellen waren nach 5 bis 7 Monaten eingeheilt und zeigten auch während der Nachbeobachtungszeit mit einer Länge von 7 Jahren keine Komplikationen [49]. Im gleichen Jahr wurde erstmals eine Finger phalanx durch ein Stück Knochen, welches mittels Tissue Engineering hergestellt worden war [50], ersetzt. Darüber hinaus wurden bereits 3 Einzelfallberichte über den Einsatz von zellbesiedelten HA-Konstrukten zur Knochenregeneration nach Tumorresektion [51] und eine Einzeldarstellung eines verheilten Knochendefekts des Radius nach dem Einsatz einer mit Wachstumsfaktoren und Zellen beladenen Matrix [52] veröffentlicht. Außerdem liegen bereits mehrere Beschreibungen des erfolgreichen Einsatzes von mit Stammzellen besiedelten Trikalziumphosphatkonstrukten zur Rekonstruktion großer Schädeldefekte $(6 \times 7 \mathrm{~cm})$ vor [53].

Eine der bekanntesten Anwendungen des Knochen-Tissue-Engineering, auch dank der Berichterstattung in der Laienpresse, stammt aus dem Bereich der Kieferchirurgie [54]. 2004 berichtete die Fachzeitschrift „The Lancet“ über die Rekonstruktion eines fast $7 \mathrm{~cm}$ langen knöchernen Defekts des Unterkiefers nach Tumorresektion mittels eines durch Tissue Engineering hergestellten Knochenersatzes. Als Scaffold diente dabei ein Titankäfig, der mit HA, Wachstumsfaktoren und MSCs beladen und danach als vaskularisiertes Transplantat nach 7 Wochen in den M. latissimus des Patienten implantiert wurde. Nach einem initial guten klinischen Ergebnis traten jedoch im weiteren Verlauf Komplikationen auf, die durch Infekte ausgelöst wurden. Außerdem erfolgte ein Bruch des Implantats. Der Patient verstarb schließlich nach etwa eineinhalb Jahren an einem kardialen Ereignis. Insgesamt liegt im Bereich der Kieferchirurgie eine umfangreichere klinische Evidenz vor als in anderen medizinischen Bereichen. So zeigte bspw. eine der seltenen randomisiert kontrollierten Studien mit insgesamt 24 Patienten eine schnellere Regeneration von 
Kieferknochendefekten durch den Einsatz von mit Stammzellen besiedelten Biomaterialien im Vergleich zur konventionellen, gelenkten Knochenregeneration [55].

Als wesentliche Limitation bei der Herstellung großer, zellbasierter Knochenkonstrukte wird die fehlende Vaskularisierung angeführt [56]. Bisher ist es noch nicht gelungen, in vitro eine funktionierende Gefäßstruktur zu züchten, geschweige denn eine solche Struktur in ein Knochenkonstrukt zu integrieren. Der Einsatz von Endothelzellen oder entsprechenden Wachstumsfaktoren soll hier Abhilfe schaffen. Jedoch wurde diese Methode bisher noch nicht in der Klinik getestet.

\section{Osteogenesis imperfecta}

Eine frühe klinische Anwendung von MSCs am Menschen war die Transplantation von MSCs bei Kindern mit schwerer Osteogenesis imperfecta. Bei unheilbaren Krankheiten mit möglicherweise letalem Ausgang ist die Schwelle zur Nutzung neuer Therapien häufig geringer, was die frühen klinischen Versuche bei dieser seltenen Erkrankung begründen mag. Bereits im Jahre 1999 wurde eine Fallserie von 3 Patienten publiziert, die eine allogene Knochenmarktransplantation erhalten hatten. Dabei wurde postuliert, dass sich die in den Transplantaten enthaltenen MSCs zu Osteoblasten differenzierten und dass ein geringer Teil dieser Zellen in den Empfängerknochen eingebaut wurde. Klinisch wurden eine Verbesserung der Knochendichte, eine gesteigerte Wachstumsrate und eine Reduktion der Frakturhäufigkeit festgestellt [57]. All diese Effekte blieben während des gesamten Beobachtungszeitraums von knapp 3 Jahren bestehen. Später wurde anhand weiterer Fallbeispiele die In-utero-Transplantation von MSCs vorgestellt [58]. Aber auch 14 Jahre später beschränken sich die Anwendungen dieser Transplantationstechnik weiterhin auf sehr kleine Studien und Fallserien [59]. Zudem zeigen neuere Ergebnisse dieser Anwendung sehr beispielhaft, wie unklar der eigentliche Wirkmechanismus der Zelltransplantation bei der Osteogenese ist: Im Knochenmark wird der überwiegende Anteil mononukleärer Zellen von nicht adhärenten Zellen gebildet, die keine MSCs im engeren Sinne darstellen. Dennoch scheinen diese Zellen als Osteoblasten im Knochen zu inkorporieren, während die eigentlichen MSCs hauptsächlich durch die Sekretion regulatorischer Substanzen und durch die Interaktion mit anderen Zellen eine positive Wirkung auf den Knochen ausüben [60]. Diese Problematik der unklaren Wirkweise von MSCs besteht auch bei anderen Anwendungen solcher Zellisolate.

\section{Knochennekrosen}

Die aseptische Knochennekrose des Femurkopfs ist wahrscheinlich jene Indikation zur Stammzelltherapie am Knochen, die bisher klinisch am besten untersucht worden ist [61]. In einer frühen Studie aus dem Jahre 2002 mit fast 200 Probanden wurde festgestellt, dass der Einsatz von Knochenmarkaspirat zu guten Ergebnissen mit einer Heilungsrate von $>90 \%$ nach durchschnittlich 7 Jahren führt, sofern die Behandlung bereits in einem frühen Stadium des Knochendefekts erfolgte [62]. Allerdings wurde diese Studie ohne Kontrollgruppe durchgeführt. Im Jahre 2008 wurde im Rahmen einer retrospektiven Untersuchung an $87 \mathrm{~Pa}-$ tienten festgestellt, dass durch die Durchführung einer Entlastungsbohrung in Kombination mit der Implantation von MSCs und dekalzifizierter Knochenmatrix eine Heilungsrate von > 75\% nach 24 Monaten erreicht und somit ein gutes Heilungsergebnis erzielt werden konnte [63] (Review in [61]). Im Jahre 2009 wurde in einer weiteren Studie gezeigt, dass die Anwendung eines konzentrierten Knochenmarkaspirats nach einer Nachbehandlungs- zeit von 27 Monaten eine Erfolgsrate von 80\% aufweist [64]. Jedoch verfügte auch diese Studie nicht über eine Kontrollgruppe. In einer neueren Studie mit 38 Probanden führte eine Dekompression mit anschließendem Einbringen eines mit MSCs und Wachstumsfaktoren beladenen Xenografts zu einer Heilungsrate von $>85 \%$ nach 4 Jahren [65]. In einer weiteren Studie mit 15 Patienten führte eine Dekompression mit Transplantation von autologem Knochen und MSCs zu einer Heilungsrate von $80 \%$ nach 24 Monaten [66].

Wenn man jedoch in der Evidenz der analysierten klinischen Studien über Level IV hinauskommen möchte, wird allerdings auch bei der Osteonekrose die Auswahl geeigneter Studien geringer. So wurden bspw. im Jahre 2004 die Ergebnisse einer randomisierten und kontrollierten Studie an 18 Gelenken publiziert, in der eine Dekompression allein mit einer Dekompression mit anschließendem Einbringen von konzentriertem Knochenmarkaspirat verglichen wurde. Nach 24 Monaten verfügte die mit Knochenmark behandelte Gruppe über eine signifikant bessere Funktion und Schmerzreduktion sowie über ein geringeres Progredienzrisiko [67]. In der vielleicht besten Studie zum Thema Osteonekrose wurde in 100 Patienten randomisiert und kontrolliert die Dekompression alleine mit der Implantation ex vivo expandierter MSCs verglichen. 60 Monate nach der OP führte die Anwendung von MSCs zu einer signifikanten Verbesserung der Funktion, zu einer wesentlichen Schmerzreduktion sowie zu einer Minimierung des Risikos eines Kollapses des Femurkopfs [68].

\section{Osteoporose}

Ob bei der Osteoporose ein Mangel an oder eine Dysfunktion der Stammzellen zur Pathogenese beiträgt, ist bislang noch nicht hinreichend untersucht worden [69]. Die Rolle der MSCs bei der Entstehung der Osteoporose sowie ihr mögliches Potenzial in der zellbasierten Krankheitstherapie wurde jedoch bereits propagiert [70-72]. Es ist bekannt, dass osteoporotische Frakturen aufgrund einer Funktionsstörung der Osteoblasten und der Osteoklasten deutlich langsamer heilen als die Frakturen gesunder Knochen. Dieser Umstand führt dazu, dass sich die Versorgung osteoporotischer Knochenbrüche sehr anspruchsvoll gestaltet [73]. Zwar sind bereits Tiermodelle für die Osteoporose verfügbar, jedoch entwickeln Versuchstiere, wie z.B. ovariektomierte Ratten oder Schafe, eine Osteoporose, die in ihrer Pathogenese der menschlichen Erkrankung nicht vollends entspricht [74]. In einer einzelnen Studie wurden bei 8 Osteoporosepatienten nach der Anwendung allogener mononukleärer Nabelschnurblutzellen erhöhte IGF-1-Serumspiegel und daraus folgend eine Verbesserung der Knochendichte beobachtet [75]. Soweit wir wissen, existieren aber bisher noch keine Studienergebnisse über die systematische Anwendung von MSCs zur Therapie der Osteoporose beim Menschen. In den kommenden Jahren muss sich die Anwendung von Stammzellen zur Knochenregeneration unter besser definierten und kontrollierten Bedingungen in randomisierten und kontrollierten Studien beweisen, um das ihr zugeschriebene Potenzial zu bestätigen [7].

\section{Knorpel \\ $\nabla$}

\section{Arthrose, traumatische Läsionen}

Die Transplantation ex vivo expandierter Gelenkchondrozyten zusammen mit einer Trägermatrix in Knorpeldefekte des Kniegelenks stellte die erste erfolgreiche Anwendung von Tissue Engi- 
neering dar [76]. Die Herstellung von Knorpelersatz aus körpereigenen Chondrozyten und einer Gelmatrix als Trägermaterial in vitro hat sich unter der Bezeichnung „matrixassistierte autologe Chondrozytenimplantation“ als erste echte Tissue-EngineeringAnwendung fest in der klinischen Praxis etabliert [73]. Diese Methode ist jedoch trotz ihrer weiten Verbreitung weiterhin umstritten. Diese Prozedur ist bei der Reparatur mehrheitlich traumatischer Knorpelläsionen jüngerer Patienten indiziert. Dabei wird ein kleines Stück Knorpel aus einem nichttragenden Bereich des Gelenks entnommen. Danach erfolgt ein Verdau des Knorpels und eine Expansion der Zellen. In einer 2. Operation werden die Zellen zusammen mit einer Matrix aus Biomaterial in den Defektbereich transplantiert [77]. Diese als (matrixgekoppelte) autologe Chondrozytenimplantation ([M]ACI) bekannte Methode wurde vor über 20 Jahren in die klinische Praxis eingeführt [78] und gilt heutzutage als etabliert. Sie ist jedoch aufgrund schlechter Langzeitergebnisse in klinischen Studien weiterhin umstritten $[79,80]$.

In den Implantaten kann es sowohl zu einer Dedifferenzierung der implantierten Chondrozyten zu einem faserigeren Knorpel als auch zu einer abschließenden Differenzierung mit Kalzifizierung kommen. Beides führt zu einer Verringerung der Gelenkfläche. Darüber hinaus müssen die Morbidität an der Entnahmestelle und die Komorbiditäten der beiden operativen Verfahren berücksichtigt werden. Die Resultate der ACI sind bei traumatischen Läsionen deutlich besser als bei degenerativen. Bei den weitaus häufiger auftretenden degenerativen osteoarthritischen Defekten ist der darunterliegende Knochen ebenfalls betroffen, wodurch das Einwachsen und die Versorgung des künstlich hergestellten Knorpels gefährdet sind. Aufgrund dieser Einschränkungen konzentrierte sich die wissenschaftliche Aufmerksamkeit in letzter Zeit ebenfalls auf die Verwendung von MSCs zur Knorpelregeneration [81-83].

In zahlreichen verschiedenen Tiermodellen wurden MSCs aus unterschiedlichen Quellen bereits genutzt, um Knorpeldefekte zu regenerieren (ausführliches Review z. B. in [84]). Eine Anwendung am Menschen ist bisher nicht über vereinzelte Fallberichte [85] und kleine Versuchsgruppen ohne Kontrollgruppe nicht hinausgekommen [86] und Berichte über systematische, kontrollierte Anwendungen fehlen in der Literatur. Doch schon eine simple Injektion von MSCs in ein arthrotisches Knie soll z. B. eine deutliche Verbesserung von Gelenkfunktion und Schmerzen bewirken [87] bzw. die schmerzfreie Laufstrecke verbessern können [88].

Ähnlich wie bei den Studien über genetische Knochenerkrankungen wurde die Effizienz von MSCs bei der Förderung der Wiederherstellung des Gelenkknorpels bereits in einigen Publikationen nachgewiesen. Dabei wurden in Kollagengel eingebettete MSCs in die Kniegelenke von Patienten mit Gelenkknorpeldefekten transplantiert [89]. Außerdem wurde gezeigt, dass die Transplantation von MSCs zu signifikanten klinischen Verbesserungen bei der Regeneration des Gelenkknorpels führen [85]. Allerdings sind die Mechanismen, die der Wiederherstellung des Knorpels zugrunde liegen, weiterhin unbekannt. Die transplantierten MSCs haben sich vielleicht zu Chondrozyten differenziert, aber es besteht ebenfalls die Möglichkeit, dass die MSCs lösliche Faktoren produzieren, mit denen sie die Differenzierung anderer Zellen aus dieser Mikroumgebung zu Gelenkknorpel induzieren. Bisher wurde jedoch noch kein routinemäßig verfügbares Handelsprodukt auf Basis dieser Methode entwickelt. Insbesondere die phänotypische Stabilität der differenzierten MSCs stellt dabei weiterhin eine große Schwierigkeit dar [90,91].

\section{Sehnen und Muskeln \\ $\nabla$}

\section{Sehnenrupturen, Tendinitis}

Ebenso wie zahlreiche andere muskuloskeletale Organe entstehen Sehnen und Muskeln aus dem Mesenchym [92]. Die embryonale Sehnenentwicklung erfolgt anfangs muskelunabhängig. Für die vollständige Entfaltung von Funktion und Form benötigt sie jedoch einen adäquaten mechanischen, zuggerichteten Stimulus $[93,94]$, sowohl durch biomechanische Zugbeanspruchung als auch durch das longitudinale Wachstum der Knochen [95]. Anschließend entwickeln sich die Muskeln aus den Muskelprogenitorzellen. Für das Tissue Engineering werden bevorzugt MSCs aus dem Knochenmark verwendet: Sie besitzen ein großes Differenzierungspotenzial, können in großen Mengen gewonnen und relativ unkompliziert aus dem Beckenkamm der Patienten entnommen werden. In tierexperimentellen Studien wurden MSCs bereits zur Defektheilung der Sehne [96] und des Muskels [97] implantiert. Bislang konnte jedoch in vitro noch keine Methode für die tenogene Differenzierung mittels Wachstumsfaktoren etabliert werden. Es ist bekannt, dass, neben BMP-12 und Scleraxis, einige Faktoren, wie z.B. PDGF-BB, TGF- $\beta$, IGF-1 oder EGF, eine entscheidende Rolle während dieser Differenzierung spielen. Allerdings ist der genaue Kommunikationsmechanismus dieser Faktoren untereinander, der zur Bildung einer funktionierenden Sehne führt, weiterhin unbekannt. In vivo zeigten erste Versuche, dass mit MSCs besiedelte Sehnengewebe scheinbar besser regenerieren, wenn sie mit Kollagen-Gel-Scaffolds kombiniert werden $[96,98]$. Neben einer besseren Sehnenregeneration führte die Verwendung von Kollagen-Scaffolds zu höheren Ausreißkräften im Vergleich zu jenen Sehnen, die nicht mit MSCs behandelt wurden $[91,98]$. Neben der Verwendung von Kollagen experimentieren verschiedene Arbeitsgruppen aktuell mit Polylaktidund Polyglykosidpolymeren, die einfach zu handhaben sind und auch in Kombination mit Electrospinning-Methoden verwendet werden können [99-101].

\section{Rechtliche Aspekte in Deutschland \\ $\nabla$}

Der Einsatz von Stammzellen zur Heilung von Patienten ist in Deutschland durch das Arzneimittelgesetz (AMG) und durch eine Vielzahl von Regularien der Europäischen Union geregelt. Eine umfangreiche Beratung zu diesem Thema kann und soll vor Verwendung dieser Zellen beim Paul-Ehrlich-Institut (www.pei.de) eingeholt werden. In erster Linie wird zwischen Arzneimitteln für neuartige Therapien, sog. „advanced therapy medicinal products“ (ATMPs), und Nicht-ATMPs unterschieden. ATMPs sind im AMG $\S 4$ b Abs. 3 geregelt und bedürfen eines speziellen Zulassungsverfahrens, bevor sie am Menschen angewendet werden dürfen. Für den täglichen Gebrauch in der Klinik sind daher die Nicht-ATMPs von besonderer Bedeutung. Zu dieser Kategorie gehören alle Zellen und Gewebe, die nicht substanziell bearbeitet worden sind. Der Begriff der substanziellen Bearbeitung ist in einem Leitfaden des Paul-Ehrlich-Instituts zur Zulassung von ATMPs geregelt [102]. Als nicht substanzielle Behandlungen von Zellen gelten das Schneiden, Zerreiben, Formen, Zentrifugieren, Einlegen in antibiotische oder antimikrobielle Lösungen, Sterilisieren, Bestrahlen, Separieren, Konzentrieren, Reinigen, Filtern, Lyophilisieren, Einfrieren und Kryopräservieren. Substanzielle Bearbeitungen sind hingegen Veränderungen des Gewebes sowie seine Kultivierung, Expansion und genetische Modifikation. Gewebe und Zellen, die auf eine der 4 vorgenannten Arten und Wei- 
sen bearbeitet wurden, gehören somit zur Gruppe der ATMPs. Somit unterliegen iPSCs und MSCs, die in der Kulturschale vermehrt werden, dem speziellen Genehmigungsverfahren für ATMPs. Außerdem sind MSCs, die für das Tissue Engineering genutzt werden, um später einem Patienten implantiert zu werden, somit ebenfalls als ATMP zu bezeichnen. Der Umgang mit Stammzellen, die von einem Spender entnommen werden, um später bei einem Patienten appliziert zu werden und die nicht zur Kategorie der ATMPs gehören, ist in AMG $\S 20 \mathrm{~b}$ und $\S 20 \mathrm{c}$ geregelt. Auch diese Zellen bedürfen eines Genehmigungsverfahrens einschließlich der Anmeldung eines von einem Arzt geleiteten Entnahmezentrums mit geeigneten Räumen sowie mit einem Herstellungs- und Qualitätsleiter. Hierbei sind die entsprechenden GMP-Richtlinien zu beachten. Diese Ausführungen betreffen bspw. ebenfalls die Etablierung einer Knochenbank. Die Anwendung von Patientenmaterial, welches unmittelbar autolog wieder im Patienten appliziert werden soll, bedarf keiner besonderen Erlaubnis, solange bspw. die Spongiosa-/Beckenkammentnahme oder das Veneninterponat den OP nicht verlässt.

\section{Interessenkonflikt: Nein}

\section{Literatur}

1 Lidgren $L$. The Bone and Joint Decade and the global economic and healthcare burden of musculoskeletal disease. J Rheumatol Suppl 2003; 67: 4-5

2 Dreinhofer KE. [The bone and joint decade-chances for orthopedics and traumatic surgery]. Z Orthop Unfall 2007; 145: 399-402

3 Sun H, Liu W, Zhou G et al. Tissue engineering of cartilage, tendon and bone. Front Med 2011; 5: 61-69

4 Brown PT, Handorf AM, Jeon WB et al. Stem cell-based tissue engineering approaches for musculoskeletal regeneration. Curr Pharm Des 2013; 19: 3429-3445

5 Colter DC, Sekiya I, Prockop DJ. Identification of a subpopulation of rapidly self-renewing and multipotential adult stem cells in colonies of human marrow stromal cells. Proc Natl Acad Sci U S A 2001; 98: 7841-7845

6 Jiang Y, Jahagirdar BN, Reinhardt RL et al. Pluripotency of mesenchymal stem cells derived from adult marrow. Nature 2002; 418: 41-49

7 Dawson JI, Kanczler J, Tare $R$ et al. Concise review: bridging the gap: bone regeneration using skeletal stem cell-based strategies-where are we now? Stem Cells 2014; 32: 35-44

8 Friedenstein AJ, Piatetzky-Shapiro II, Petrakova KV. Osteogenesis in transplants of bone marrow cells. J Embryol Exp Morphol 1966; 16: 381-390

9 Zomorodian E, Baghaban Eslaminejad M. Mesenchymal stem cells as a potent cell source for bone regeneration. Stem Cells Int 2012; 2012: 980353

10 Pittenger MF, Mackay AM, Beck SC et al. Multilineage potential of adult human mesenchymal stem cells. Science 1999; 284: 143-147

11 Kио TK, Ho JH, Lee OK. Mesenchymal stem cell therapy for nonmusculoskeletal diseases: emerging applications. Cell Transplant 2009; 18: 1013-1028

12 Bruder SP, Ricalton NS, Boynton RE et al. Mesenchymal stem cell surface antigen SB-10 corresponds to activated leukocyte cell adhesion molecule and is involved in osteogenic differentiation. J Bone Miner Res 1998; 13: 655-663

13 Haynesworth SE, Baber MA, Caplan AI. Cell surface antigens on human marrow-derived mesenchymal cells are detected by monoclonal antibodies. Bone 1992; 13: 69-80

14 Dominici M, Le Blanc K, Mueller I et al. Minimal criteria for defining multipotent mesenchymal stromal cells. The International Society for Cellular Therapy position statement. Cytotherapy 2006; 8: 315-317

15 Augello A, Tasso R, Negrini SM et al. Bone marrow mesenchymal progenitor cells inhibit lymphocyte proliferation by activation of the programmed death 1 pathway. Eur J Immunol 2005; 35: 1482-1490

16 Kode JA, Mukherjee S, Joglekar MV et al. Mesenchymal stem cells: immunobiology and role in immunomodulation and tissue regeneration. Cytotherapy 2009; 11: 377-391
17 Kolar P, Schmidt-Bleek $K$, Schell $H$ et al. The early fracture hematoma and its potential role in fracture healing. Tissue Eng Part B Rev 2010; 16: 427-434

18 Chatterjea A, Lapointe VL, Alblas J et al. Suppression of the immune system as a critical step for bone formation from allogeneic osteoprogenitors implanted in rats. J Cell Mol Med 2014; 18: 134-142

19 Evans MJ, Kaufman MH. Establishment in culture of pluripotential cells from mouse embryos. Nature 1981; 292: 154-156

20 Thomson JA, Itskovitz-Eldor J, Shapiro SS et al. Embryonic stem cell lines derived from human blastocysts. Science 1998; 282: 1145-1147

21 Takahashi K, Yamanaka S. Induction of pluripotent stem cells from mouse embryonic and adult fibroblast cultures by defined factors. Cell 2006; 126: 663-676

$22 \mathrm{Yu}$ J, Vodyanik MA, Smuga-Otto $\mathrm{K}$ et al. Induced pluripotent stem cell lines derived from human somatic cells. Science 2007; 318: 19171920

$23 \mathrm{Yu}$ J, Hu K, Smuga-Otto K et al. Human induced pluripotent stem cells free of vector and transgene sequences. Science 2009; 324: 797-801

24 Walmsley GG, Hyun J, McArdle A et al. Induced pluripotent stem cells in regenerative medicine and disease modeling. Curr Stem Cell Res Ther 2014; 9: 73-81

$25 \mathrm{Kim}$ N, Cho SG. Clinical applications of mesenchymal stem cells. Korean J Intern Med 2013; 28: 387-402

26 Stolzing A, Jones E, McGonagle D et al. Age-related changes in human bone marrow-derived mesenchymal stem cells: consequences for cell therapies. Mech Ageing Dev 2008; 129: 163-173

27 Li Z, Liu C, Xie Z et al. Epigenetic dysregulation in mesenchymal stem cell aging and spontaneous differentiation. PLoS One 2011; 6: e20526

28 Liu L, Rando TA. Manifestations and mechanisms of stem cell aging. J Cell Biol 2011; 193: 257-266

29 Yan X, Ehnert S, Culmes M, et al. 5-azacytidine improves the osteogenic differentiation potential of aged human adipose-derived mesenchymal stem cells by DNA demethylation. PLoS One 2014; 9: e90846

30 Homma Y, Zimmermann G, Hernigou P. Cellular therapies for the treatment of non-union: the past, present and future. Injury 2013; 44 (Suppl. 1): S46-S49

31 Connolly JF, Guse R, Tiedeman J et al. Autologous marrow injection for delayed unions of the tibia: a preliminary report. J Orthop Trauma 1989; 3: 276-282

32 Connolly JF, Guse R, Tiedeman J et al. Autologous marrow injection as a substitute for operative grafting of tibial nonunions. Clin Orthop Relat Res 1991; 266: 259-270

33 Hernigou P, Poignard A, Beaujean $F$ et al. Percutaneous autologous bone-marrow grafting for nonunions. Influence of the number and concentration of progenitor cells. J Bone Joint Surg Am 2005; 87: $1430-1437$

$34 \mathrm{Kim}$ SJ, Shin YW, Yang KH et al. A multi-center, randomized, clinical study to compare the effect and safety of autologous cultured osteoblast(Ossron) injection to treat fractures. BMC Musculoskelet Disord 2009; $10: 20$

35 Dreger T, Watson JT, Akers $W$ et al. Intravenous application of CD271selected mesenchymal stem cells during fracture healing. J Orthop Trauma 2014; 28 (Suppl. 1): S15-S19

36 Fayaz HC, Giannoudis PV, Vrahas MS et al. The role of stem cells in fracture healing and nonunion. Int Orthop 2011; 35: 1587-1597

37 Cancedda R, Giannoni P, Mastrogiacomo M. A tissue engineering approach to bone repair in large animal models and in clinical practice. Biomaterials 2007; 28: 4240-4250

38 Pneumaticos SG, Triantafyllopoulos GK, Basdra EK et al. Segmental bone defects: from cellular and molecular pathways to the development of novel biological treatments. J Cell Mol Med 2010; 14: 2561-2569

39 Pape HC, Evans A, Kobbe P. Autologous bone graft: properties and techniques. J Orthop Trauma 2010; 24 (Suppl. 1): S36-S40

40 Kanellopoulos $A D$, Soucacos PN. Management of nonunion with distraction osteogenesis. Injury 2006; 37 (Suppl. 1): S51-S55

41 Mistry AS, Mikos AG. Tissue engineering strategies for bone regeneration. Adv Biochem Eng Biotechnol 2005; 94: 1-22

42 Giannoudis PV, Einhorn TA, Marsh D. Fracture healing: the diamond concept. Injury 2007; 38 (Suppl. 4): S3-S6

43 Havens AM, Shiozawa Y, Jung Y et al. Human very small embryonic-like cells generate skeletal structures, in vivo. Stem Cells Dev 2013; 22: 622-630

44 Berner A, Reichert JC, Woodruff MA et al. Autologous vs. allogenic mesenchymal progenitor cells for the reconstruction of critical sized 
segmental tibial bone defects in aged sheep. Acta Biomater 2013; 9: 7874-7884

45 Lee JW, Kim KJ, Kang KS et al. Development of a bone reconstruction technique using a solid free-form fabrication (SFF)-based drug releasing scaffold and adipose-derived stem cells. J Biomed Mater Res A 2013; 101: 1865-1875

$46 \mathrm{Du} \mathrm{CH}, \mathrm{Li} \mathrm{NY}$, Gao $\mathrm{N}$ et al. A preliminary study on the application of bone marrow stromal cell sheet on the formation of functional tissueengineered bone in dogs. J Oral Maxillofac Surg 2013; 71: 1531.e11531.e10

47 Meijer GJ, de Bruijn JD, Koole R et al. Cell based bone tissue engineering in jaw defects. Biomaterials 2008; 29: 3053-3061

48 Quarto $R$, Mastrogiacomo $M$, Cancedda $R$ et al. Repair of large bone defects with the use of autologous bone marrow stromal cells. N Engl J Med 2001; 344: 385-386

49 Marcacci M, Kon E, Moukhachev V et al. Stem cells associated with macroporous bioceramics for long bone repair: 6- to 7-year outcome of a pilot clinical study. Tissue Eng 2007; 13: 947-955

50 Vacanti CA, Bonassar LJ, Vacanti MP et al. Replacement of an avulsed phalanx with tissue-engineered bone. N Engl J Med 2001; 344: 15111514

51 Morishita T, Honoki K, Ohgushi H et al. Tissue engineering approach to the treatment of bone tumors: three cases of cultured bone grafts derived from patients' mesenchymal stem cells. Artif Organs 2006; 30: 115-118

52 Calori GM, Mazza E, Colombo M et al. The use of bone-graft substitutes in large bone defects: any specific needs? Injury 2011; 42 (Suppl. 2): S56-S63

53 Thesleff T, Lehtimaki K, Niskakangas T et al. Cranioplasty with adiposederived stem cells and biomaterial: a novel method for cranial reconstruction. Neurosurgery 2011; 68: 1535-1540

54 Gronthos $S$. Reconstruction of human mandible by tissue engineering. Lancet 2004; 364: 735-736

55 Kaigler D, Pagni G, Park CH et al. Stem cell therapy for craniofacial bone regeneration: a randomized, controlled feasibility trial. Cell Transplant 2013; 22: 767-777

56 Buckwalter JA, Glimcher MJ, Cooper RR et al. Bone biology. I: Structure, blood supply, cells, matrix, and mineralization. Instr Course Lect 1996; 45: 371-386

57 Horwitz EM, Prockop DJ, Fitzpatrick LA et al. Transplantability and therapeutic effects of bone marrow-derived mesenchymal cells in children with osteogenesis imperfecta. Nat Med 1999; 5: 309-313

58 Le Blanc K, Götherström C, Ringdén $O$ et al. Fetal mesenchymal stem-cell engraftment in bone after in utero transplantation in a patient with severe osteogenesis imperfecta. Transplantation 2005; 79: 1607-1614

59 Götherström C, Westgren M, Shaw SW et al. Pre- and postnatal transplantation of fetal mesenchymal stem cells in osteogenesis imperfecta: a two-center experience. Stem Cells Transl Med 2014; 3: 255-264

60 Otsuru S, Gordon PL, Shimono Ket al. Transplanted bone marrow mononuclear cells and MSCs impart clinical benefit to children with osteogenesis imperfecta through different mechanisms. Blood 2012; 120 : 1933-1941

61 Hauzeur JP, Gangji V. Phases 1-3 clinical trials using adult stem cells in osteonecrosis and nonunion fractures. Stem Cells Int 2010; 2010: 410170

62 Hernigou P, Beaujean F. Treatment of osteonecrosis with autologous bone marrow grafting. Clin Orthop Relat Res 2002; 405: 14-23

63 Ji WF, Ding WH, Ma ZC et al. [Three-tunnels core decompression with implantation of bone marrow stromal cells (bMSCs) and decalcified bone matrix (DBM) for the treatment of early femoral head necrosis]. Zhongguo Gu Shang 2008; 21: 776-778

64 Wang BL, Sun W, Shi ZC et al. Treatment of nontraumatic osteonecrosis of the femoral head with the implantation of core decompression and concentrated autologous bone marrow containing mononuclear cells. Arch Orthop Trauma Surg 2010; 130: 859-865

65 Calori GM, Mazza E, Colombo M et al. Treatment of AVN using the induction chamber technique and a biological-based approach: Indications and clinical results. Injury 2014; 45: 369-373

66 Wang T, Wang W, Yin ZS. Treatment of osteonecrosis of the femoral head with thorough debridement, bone grafting and bone-marrow mononuclear cells implantation. Eur J Orthop Surg Traumatol 2014; 24: 197-202

67 Gangji V, Hauzeur JP, Matos C et al. Treatment of osteonecrosis of the femoral head with implantation of autologous bone-marrow cells. A pilot study. J Bone Joint Surg Am 2004; 86: 1153-1160
68 Zhao D, Cui D, Wang B et al. Treatment of early stage osteonecrosis of the femoral head with autologous implantation of bone marrow-derived and cultured mesenchymal stem cells. Bone 2012; 50: 325-330

69 Dalle Carbonare L, Valenti MT, Zanatta M et al. Circulating mesenchymal stem cells with abnormal osteogenic differentiation in patients with osteoporosis. Arthritis Rheum 2009; 60: 3356-3365

70 Antebi B, Pelled G, Gazit D. Stem cell therapy for osteoporosis. Curr Osteoporos Rep 2014; 12: 41-47

71 Peng S, Zhou G, Luk KD et al. Strontium promotes osteogenic differentiation of mesenchymal stem cells through the Ras/MAPK signaling pathway. Cell Physiol Biochem 2009; 23: 165-174

72 Cho SW, Sun HJ, Yang JY et al. Transplantation of mesenchymal stem cells overexpressing RANK-Fc or CXCR4 prevents bone loss in ovariectomized mice. Mol Ther 2009; 17: 1979-1987

73 Egermann $M$, Schneider E, Evans $\mathrm{CH}$ et al. The potential of gene therapy for fracture healing in osteoporosis. Osteoporos Int 2005; 16 (Suppl. 2): S120-S128

74 Lelovas PP, Xanthos TT, Thoma SE et al. The laboratory rat as an animal model for osteoporosis research. Comp Med 2008; 58: 424-430

75 Li J, Zhang L, Zhou L et al. Beneficial effects of non-matched allogeneic cord blood mononuclear cells upon patients with idiopathic osteoporosis. J Transl Med 2012; 10: 102

76 Moreira-Teixeira LS, Georgi N, Leijten J et al. Cartilage tissue engineering. Endocr Dev 2011; 21: 102-115

77 Ducheyne P, Mauck RL, Smith DH. Biomaterials in the repair of sports injuries. Nat Mater 2012; 11: 652-654

78 Brittberg $M$, Lindahl A, Nilsson A et al. Treatment of deep cartilage defects in the knee with autologous chondrocyte transplantation. N Engl J Med 1994; 331: 889-895

79 Kon E, Filardo G, Di Martino A et al. ACI and MACI. J Knee Surg 2012; 25: $17-22$

80 Iwasa J, Engebretsen L, Shima Y et al. Clinical application of scaffolds for cartilage tissue engineering. Knee Surg Sports Traumatol Arthrosc 2009; 17: 561-577

81 Castro NJ, Hacking SA, Zhang LG. Recent progress in interfacial tissue engineering approaches for osteochondral defects. Ann Biomed Eng 2012: 40: 1628-1640

82 Dhinsa BS, Adesida $A B$. Current clinical therapies for cartilage repair, their limitation and the role of stem cells. Curr Stem Cell Res Ther 2012; 7: 143-148

83 Huselstein C, Li Y, He X. Mesenchymal stem cells for cartilage engineering. Biomed Mater Eng 2012; 22: 69-80

84 Deng S, Huang $R$, Wang J et al. Miscellaneous animal models accelerate the application of mesenchymal stem cells for cartilage regeneration. Curr Stem Cell Res Ther 2014; 9: 223-233

85 Centeno CJ, Busse D, Kisiday J et al. Increased knee cartilage volume in degenerative joint disease using percutaneously implanted, autologous mesenchymal stem cells. Pain Physician 2008; 11: 343-353

86 Sekiya I, Muneta T, Koga $\mathrm{H}$ et al. [Articular cartilage regeneration with synovial mesenchymal stem cells]. Clin Calcium 2011; 21: 879-889

87 Orozco L, Munar A, Soler R et al. Treatment of knee osteoarthritis with autologous mesenchymal stem cells: a pilot study. Transplantation 2013; 95: 1535-1541

88 Davatchi F, Abdollahi BS, Mohyeddin $M$ et al. Mesenchymal stem cell therapy for knee osteoarthritis. Preliminary report of four patients. Int J Rheum Dis 2011; 14: 211-215

89 Gobbi A, Karnatzikos G, Sankineani SR. One-step surgery with multipotent stem cells for the treatment of large full-thickness chondral defects of the knee. Am J Sports Med 2014; 42: 648-657

90 Pelttari $K$, Steck E, Richter $W$. The use of mesenchymal stem cells for chondrogenesis. Injury 2008; 39 (Suppl. 1): S58-S65

91 Randau TM, Schildberg FA, Alini $M$ et al. The effect of dexamethasone and triiodothyronine on terminal differentiation of primary bovine chondrocytes and chondrogenically differentiated mesenchymal stem cells. PLoS One 2013; 8: e72973

92 Sadler TW, Langman J, Drews U. Medizinische Embryologie - die normale menschliche Entwicklung und ihre Fehlbildungen. 11. Aufl. Stuttgart: Thieme; 2008

93 Asou Y, Nifuji A, Tsuji K et al. Coordinated expression of scleraxis and Sox9 genes during embryonic development of tendons and cartilage. J Orthop Res 2002; 20: 827-833

94 Tozer S, Duprez D. Tendon and ligament: development, repair and disease. Birth Defects Res C Embryo Today 2005; 75: 226-236

95 Benjamin $M$, Ralphs JR. The cell and developmental biology of tendons and ligaments. Int Rev Cytol 2000; 196: 85-130 
96 Awad HA, Butler DL, Boivin GP et al. Autologous mesenchymal stem cell-mediated repair of tendon. Tissue Eng 1999; 5: 267-277

97 Saito T, Dennis JE, Lennon DP et al. Myogenic expression of mesenchymal stem cells within myotubes of mdx mice in vitro and in vivo. Tissue Eng 1995; 1: 327-343

98 Awad HA, Boivin GP, Dressler MR et al. Repair of patellar tendon injuries using a cell-collagen composite. J Orthop Res 2003; 21: 420-431

99 Shih YR, Chen CN, Tsai SW et al. Growth of mesenchymal stem cells on electrospun type I collagen nanofibers. Stem Cells 2006; 24: 2391-2397
100 Zhang YZ, Venugopal J, Huang ZM et al. Characterization of the surface biocompatibility of the electrospun PCL-collagen nanofibers using fibroblasts. Biomacromolecules 2005; 6: 2583-2589

101 Martins A, Reis RL, Neves NM. Electrospinning: processing technique for tissue engineering scaffolding. Int Mater Rev 2008; 53: 257-274

102 Paul-Ehrlich Institut. Arzneimittel für neuartige Therapien ATMP Regulatorische Anforderungen und Praktische Hinweise. Im Internet: http://www.pei.de/SharedDocs/Downloads/pu/innovationsbuero/ broschuere-atmp-anforderungen-hinweise.pdf; Stand: 2012 